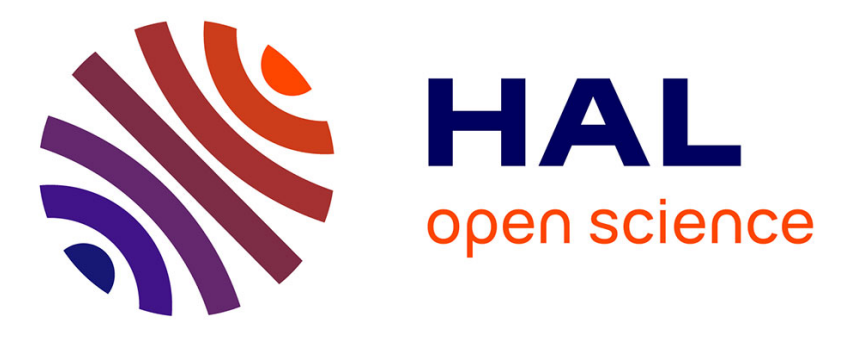

\title{
Decreasing Phanerozoic extinction intensity as a consequence of Earth surface oxygenation and metazoan ecophysiology
}

Richard Stockey, Alexandre Pohl, Andy Ridgwell, Seth Finnegan, Erik Sperling

\section{To cite this version:}

Richard Stockey, Alexandre Pohl, Andy Ridgwell, Seth Finnegan, Erik Sperling. Decreasing Phanerozoic extinction intensity as a consequence of Earth surface oxygenation and metazoan ecophysiology. Proceedings of the National Academy of Sciences of the United States of America, 2021, 118 (41), pp.e2101900118. hal-03364856

\section{HAL Id: hal-03364856 https://hal.science/hal-03364856}

Submitted on 6 Oct 2021

HAL is a multi-disciplinary open access archive for the deposit and dissemination of scientific research documents, whether they are published or not. The documents may come from teaching and research institutions in France or abroad, or from public or private research centers.
L'archive ouverte pluridisciplinaire HAL, est destinée au dépôt et à la diffusion de documents scientifiques de niveau recherche, publiés ou non, émanant des établissements d'enseignement et de recherche français ou étrangers, des laboratoires publics ou privés. 


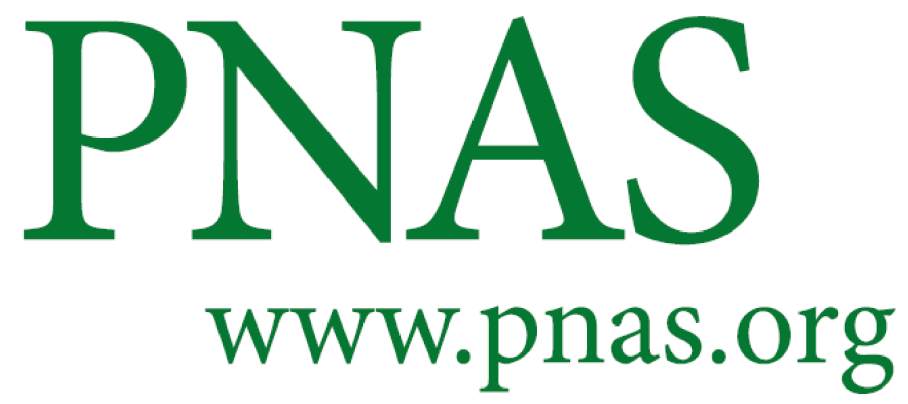

\section{Main Manuscript for}

\section{Decreasing Phanerozoic extinction intensity as a consequence of Earth surface oxygenation and metazoan ecophysiology}

Richard G. Stockey ${ }^{1 *}$, Alexandre Pohl ${ }^{2,3}$, Andy Ridgwell ${ }^{2}$, Seth Finnegan ${ }^{4}$, and Erik A. Sperling ${ }^{1}$

${ }^{1}$ Department of Geological Sciences, Stanford University, Stanford, CA, USA

${ }^{2}$ Department of Earth and Planetary Sciences, University of California, Riverside, CA, USA

${ }^{3}$ Biogéosciences, UMR 6282, UBFC/CNRS, Université Bourgogne Franche-Comté, 6 boulevard Gabriel, F-21000 Dijon, France

${ }^{4}$ Department of Integrative Biology, University of California, Berkeley, Berkeley, CA, USA

${ }^{*}$ To whom correspondence should be addressed.

Email: Richard Stockey (rstockey@stanford.edu)

Author Contributions: R.G.S. and E.A.S. designed the research; R.G.S. conducted Earth system modeling with input from A.R.; A.P. and A.R. generated the Ordovician model configuration; R.G.S. conducted ecophysiological modeling; R.G.S. and S.F. conducted quantitative paleobiological analyses; R.G.S wrote the paper with contributions from all authors.

Classification: Physical Sciences/Earth, Atmospheric, and Planetary Sciences

Keywords: Extinction, oxygen, ecophysiology, temperature-dependent hypoxia, Earth system evolution

\section{This PDF file includes:}

Main Text

Figure Captions 


\section{Abstract}

2 The decline in background extinction rates of marine animals through geologic time is an

3 established but unexplained feature of the Phanerozoic fossil record. There is also

4 growing consensus that the ocean and atmosphere did not become oxygenated to near-

5 modern levels until the mid-Paleozoic, coinciding with the onset of generally lower

6 extinction rates. Physiological theory provides us with a possible causal link between

7 these two observations - predicting that the synergistic impacts of oxygen and

8 temperature on aerobic respiration would have made marine animals more vulnerable to

9 ocean warming events during periods of limited surface oxygenation. Here, we evaluate

10 the hypothesis that changes in surface oxygenation exerted a first-order control on

11 extinction rates through the Phanerozoic using a combined Earth system and

12 ecophysiological modeling approach. We find that although continental configuration, the

13 efficiency of the biological carbon pump in the ocean, and initial climate state all impact

14 the magnitude of modeled biodiversity loss across simulated warming events,

15 atmospheric oxygen is the dominant predictor of extinction vulnerability, with metabolic

16 habitat viability and global ecophysiotype extinction exhibiting inflection points around

$1740 \%$ of present atmospheric oxygen. Given this is the broad upper limit for estimates of

18 early Paleozoic oxygen levels, our results are consistent with the relative frequency of

19 high-magnitude extinction events (particularly those not included in the canonical Big

20 Five mass extinctions) early in the Phanerozoic being a direct consequence of limited

21 early Paleozoic oxygenation and temperature-dependent hypoxia responses.

\section{Significance Statement}

24 The decline in extinction rates through geologic time is a well-established but enigmatic

25 feature of the marine animal fossil record. We hypothesize that this trend is driven largely

26 by secular changes in the oxygenation of the atmosphere and oceans, as physiological

27 principles predict that marine animals would have been more vulnerable to ocean

28 warming during intervals of geological time with limited atmospheric oxygenation. We 
test this at a global oceanographic scale by combining models of ocean biogeochemistry and animal physiology. We show that atmospheric oxygen exerts a first-order control on the simulated extinction vulnerability of marine animals, highlighting its likely importance in controlling extinction trends through geologic time.

\section{Introduction}

35 One of the most striking and poorly understood features of the marine fossil record is a 36 long-term decline in apparent extinction rates through the Phanerozoic (1-7). First 37 revealed by analyses of stratigraphic range compilations $\sim 40$ years ago (1), this decline 38 has been a persistent feature in subsequent analyses using expanded databases (8) and 39 improved extinction rate and richness metrics since then (e.g. (2-7); Figs. 1, S1).

40 Declining genus-level extinction rates are a consistent feature of Phanerozoic analyses 41 using a broad range of different richness and extinction rate metrics ((7); Fig. S2, Table $42 \mathrm{~S} 1$ ), and are not substantially altered by varying temporal binning or controlling for 43 species/genus ratio (Figs. S1-2, Table S1; Materials and Methods). In particular, the 44 Cambrian and Ordovician Periods stand out as intervals of especially high faunal 45 turnover $((6,7,9)$; Figs. S1-2), to the point that the early Paleozoic is often treated 46 separately from the rest of the Phanerozoic in quantitative analyses of extinction (e.g. (7, $4710,11)$ ). Changes in the distribution of lineage ages and geographic range sizes, area of 48 continental shelf environments, intensity of species interactions, frequency of geological triggers, stabilization of Earth's climate, and rock-area sampling biases have all been 50 proposed as drivers of this trend (10-14). However, despite the long history of study and 51 numerous proposed explanations, no consensus has been reached regarding the drivers of 52 much higher early Paleozoic extinction rates vs. those of the later Paleozoic, Mesozoic 53 and Cenozoic.

Secular trends in the oxygenation of the ocean and atmosphere are an underexplored factor that may help explain changing extinction intensity through time. A

56 growing body of geochemical proxy evidence suggests that Earth's surface did not

57 become oxygenated to near-modern levels until the early-mid Paleozoic (Fig. 1) (15-20) 
and the most recent generation of long-term carbon cycle models support only limited early Paleozoic oxygenation (21-23). While early Paleozoic marine oxygen concentrations were self-evidently above minimum thresholds for aerobic metazoan metabolism (at least, in the shallow ocean), modern observations clearly show that variations in dissolved oxygen exert a strong control on marine biodiversity (24-26).

63 Moreover, experimental respirometry, metabolic theory, and ecophysiological modeling have demonstrated that the synergistic effects of oxygen and temperature on capacity for aerobic respiration significantly influence the metabolic viability of marine habitat (2732). Following the principle of oxygen and capacity limited thermal tolerance (OCLTT see Pörtner (28) for review), the critical partial pressure of oxygen $\left(p \mathrm{O}_{2}\right)$ required to sustain resting metabolism increases exponentially with temperature, and the increase in oxygen supply required for ecologically sustainable populations results in a further steepening of this exponential relationship (Fig. 2; (33)). Meta-analyses investigating the sensitivity of modern marine ectotherms to climate-related stressors and the vulnerability of their fossil representatives to extinction further suggest that physiological traits of modern marine animals can be generally scaled to extinction sensitivity to ancient climate perturbations (34). system with limited surface oxygenation, leading to the prediction that marine ectotherms would have been substantially more vulnerable to temperature-dependent hypoxia, and therefore climate-driven extinction, due to reduced thermal safety margins in poorly oxygenated ancient oceans (Fig. 2; (35)). This logic further leads to the implication that dissolved oxygen was likely important to the structure of marine ecosystems at concentrations well above critical hypoxic thresholds that have previously been invoked for the evolution of early animals $(36,37)$, as is observed at regional scales in modern marine environments $(29-31,38)$. Recent advances in Earth system modeling provide an opportunity to test this hypothesis at the planetary-scale and to explicitly distinguish the role of changes in surface oxygenation through the Phanerozoic from variations in continental configuration, biological carbon cycling and climate state. 
Our approach is two-fold - incorporating both Earth system and ecophysiological modeling. First, using an Earth system model of intermediate complexity (cGENIE (39) see Materials and Methods and Supplementary Information) and an ensemble experimental approach, we generate a suite of three-dimensional realizations of potential marine environmental conditions. These Earth system model experiments span a range of

92 scenarios for atmospheric $\mathrm{O}_{2}$ and $\mathrm{CO}_{2}$ concentrations (Fig. 3a) in order to address uncertainty associated with reconstructions of these variables through geologic time (e.g.

Fig 1b). Atmospheric $\mathrm{CO}_{2}$ concentrations are varied to force global climate in our experiments and solar forcing is kept constant for each ensemble (Fig. 3). Simulating the potential three-dimensional structure of past oceans in these experiments is critical: in the upper ocean, oxygen and temperature both decrease with depth, but have opposite gradients with respect to latitude $(33,38)$. Temperature gradients decrease towards the poles, while subsurface oxygen gradients increase. Because aerobic habitability for marine animals involves the synergistic combination of oxygen and temperature $(29,34$, 35,38 ), elucidating the spatial complexity of the environmental landscape of ancient oceans is required to appropriately understand biological responses to environmental perturbations.

We additionally identify key boundary conditions that have significantly changed through Earth history and may offer alternative explanations for the trends in extinction rates observed in the fossil record. 1) Continental configuration, which affects latitudinal habitat (i.e. continental shelf) distribution, as well as ocean circulation (and resulting temperature and oxygen gradients), and could therefore have modulated the susceptibility of animals to extinction $(33,40,41)$. 2) The efficiency of the biological carbon pump in the ocean, which exerts a control on the distribution of oxygen in the upper water column (42) and has been previously identified as important in differentiating earlier Paleozoic vs. Mesozoic-Cenozoic ocean redox conditions (17). 3) Climate state, which directly impacts ocean surface temperatures (and by extension dissolved marine oxygen) and therefore influences how close animals at a given latitude are living to their physiological limits $(29,38)$. 
Utilizing model ocean temperatures and dissolved oxygen concentrations from

117 this suite of 3D realizations, we then employ the recently developed Metabolic Index (29,

11833,38 ) in order to evaluate the proportion of modern marine ectotherms (based upon available experimental respirometry data) that could survive in these simulated environments. While we do not attempt to directly simulate taxonomic diversity, the

121 Metabolic Index provides us with a means to mechanistically assess the physiological ecotypes (herein, ecophysiotypes) that can contribute to biodiversity. We apply a probabilistic representation of temperature-dependent hypoxia responses (Fig. 2) to

124 define 'metabolic habitat viability', a global metric describing the percentage of feasible ecophysiotypes that can live anywhere in the shelf environments of our 3D ocean models.

126 Following other paleoecological modeling approaches (e.g. (41)), we focus our analyses

127 on shelf environments because they constitute the majority of the Phanerozoic fossil record and host the majority of marine biodiversity in the modern, although we provide complimentary simulations for the entire global ocean.

Finally, we estimate global extinction sensitivity across a range of atmospheric $\mathrm{O}_{2}$ concentrations to link our ecophysiological models more directly to the trends we observe

132 in the geologic record. We model 'global ecophysiotype extinction' as the predicted loss

133 of ecophysiotypes across a nominal warming event of $\sim 5^{\circ} \mathrm{C}$ (as defined by mean

134 temperature change in the equatorial surface ocean; Fig. S3, Table S2). These warming

135 events are represented by Earth system models separated by two doublings of

136 atmospheric $\mathrm{CO}_{2}$, and are designed to mimic relatively extreme (but not infrequent)

137 examples of climate variability known to have occurred throughout Earth history (43,

138 44). We performed this extinction sensitivity estimate for all scenarios of atmospheric $\mathrm{O}_{2}$

139 and for each ensemble (continental configuration, biological pump efficiency; Figs. 3,

140 S3) across multiple baseline climate states. We further explore the consistency of our

141 results across different thresholds for minimum habitat area (number of equal area ocean

142 model cells) and tolerances for proportional habitat loss, as well as across deviations from

143 the distributions of physiological tolerances observed in modern marine ectotherms.

144 Thus, by combining ecophysiology and ensemble Earth system modeling, we evaluate the 145 vulnerability of marine ectotherms to warming events at a range of atmospheric $\mathrm{O}_{2}$ levels 
146 in a framework that is neutral to the selective advantage of different ecophysiotypes and

147 explores the relative impacts of other aspects of Phanerozoic Earth system evolution on 148 marine animal ecophysiology.

150 Results

Metabolic habitat viability

Metabolic habitat viability - the total number of ecophysiotypes that can live in the shelf environments of each ancient ocean realization - is shown in Figure 4 for our ensemble of simulations employing an Ordovician continental configuration and Figure S4 for all other Earth system model ensembles. In all ensembles, metabolic habitat viability exhibits a positive relationship with atmospheric $\mathrm{O}_{2}$ and a negative relationship with global temperature, resulting in a sigmoidal relationship between atmospheric $\mathrm{O}_{2}$ and metabolic habitat viability for a given climate state. While our primary analyses focus on shelf environments, very similar trends are observed when the entire ocean is considered as potential habitat for marine animals (Figs. S5-6). Using entire 3D ocean models often results in slightly higher estimates of metabolic habitat viability because cooler and (at higher atmospheric $\mathrm{O}_{2}$ levels) well-oxygenated deep ocean environments may act as refugia, despite being unlikely diversity hotspots for other reasons such as food supply $(45,46)$. The latitudinal distribution of viable ecophysiotypes is similar between continental configurations (Figs. 3, S3), with more ecophysiotypes generally able to inhabit cooler polar regions of the surface ocean. Identifying oceanographic and tectonic drivers of regional differences in viable ecophysiotype biogeography between continental configurations is an interesting avenue for future research, but this would require tuning model ensembles to precisely equivalent climate states and is therefore beyond the scope of this study.

Changes in metabolic habitat viability between cooler and warmer climate states allow us to begin linking ecophysiology to extinction sensitivity under different scenarios of atmospheric oxygenation (Fig. 4). The percentage change in metabolic habitat viability 
174 between colder and warmer ocean simulations increases as atmospheric oxygen decreases 175 from modern levels (100\% preindustrial atmospheric levels, PAL), reaching maximum 176 values at atmospheric $\mathrm{O}_{2}$ concentrations reconstructed for the early Paleozoic $(<40-50 \%$ 177 PAL - grey envelope in Figs. 4, S4). Furthermore, because extinction is expected to track 178 the proportional rather than absolute loss of ecophysiotypes $(33,40)$, differences in 179 metabolic habitat viability would therefore be amplified to result in high levels of 180 warming-driven extinction at lower levels of atmospheric oxygenation reconstructed for 181 the early Paleozoic (e.g. 20\% PAL). In such cases, the number of viable ecophysiotypes 182 in any low- $\mathrm{O}_{2}$ scenario is much lower, leading to greater proportional change despite a 183 similar absolute change in metabolic habitat viability to that predicted at higher $\mathrm{O}_{2}$ levels 184 (e.g. 80\% PAL). Our analyses thus demonstrate that the expected proportional loss of 185 ecophysiotypes during a global warming event would be substantially higher under the 186 limited surface oxygenation scenarios hypothesized for the early Paleozoic than for 187 modern (or, more broadly, post-mid-Paleozoic) levels of oxygenation.

When we simulate the impacts of warming events on the extinction of marine animals, the general trend of greater predicted extinction at lower atmospheric oxygen 192 levels is a common feature of all ensemble experiments (Figs. 5, S7-12). There is 193 variation in the absolute magnitude of simulated global ecophysiotype extinction between 194 the different continental configurations tested in our ensembles. However, we do not 195 observe any time-dependent trends in extinction sensitivity between progressive 196 continental reconstructions and the most striking feature of the results remains the non197 linear increase in global ecophysiotype extinction with declining oxygenation (Figs. 5, $198 \mathrm{~S} 7-12$ ). Below 10\% PAL $\mathrm{O}_{2}$, the relationship between modeled extinction and 199 atmospheric oxygen is less predictable. However, the initial pool of viable pre-extinction 200 ecophysiotypes in these simulations is very small at low $\mathrm{O}_{2}$ levels (Figs. 3, 4, S4-6), 201 suggesting that this inconsistency may partially be a product of sample size (as supported 202 by wider envelopes in Figures 5, S7-8, S11) and the limited experimental respirometry 
data for animals that live in low-oxygen environments (47). We find that modeled extinction is high at low atmospheric oxygen levels for the Ordovician configuration regardless of the efficiency of the biological pump (Fig. S7), suggesting that despite its role in controlling the distribution of oxygen in the upper water column this factor is of secondary importance compared to atmospheric $\mathrm{O}_{2}$. A shallower remineralization depth does slightly reduce simulated extinction sensitivity at low atmospheric $\mathrm{O}_{2}(\sim 5-20 \%$ PAL), although the same caveat of small initial ecophysiotype population size applies.

Increasing the baseline temperature in our extinction simulations (i.e. testing for any initial climate state dependence of extinction susceptibility), results in increased predicted global ecophysiotype extinction for all three continental configurations (Fig. S8). A corresponding decrease in modeled ecophysiotype extinction is generally observed at cooler baseline temperatures. Cool, low $\mathrm{O}_{2}(<20 \% \mathrm{PAL})$ Ordovician simulations are a minor exception to this general rule, suggesting that continental configuration may exert a second-order control on the climate state dependence of extinction vulnerability. The impact of initial climate state on simulated ecophysiotype extinction appears less dramatic than the impact of atmospheric oxygen, particularly in light of the apparent inflection in modeled extinction observed around upper estimates for early Paleozoic $\mathrm{O}_{2}$ levels. However, we note that pre-extinction climate state impacts modeled ecophysiotype extinction more than any other Earth system boundary condition explored in this study.

Altering the assumptions of our ecophysiological modeling approach does not dramatically impact the observed relationship between predicted extinction and atmospheric oxygen. In our primary analyses, we define ecophysiotype extinction as only occurring when no cells in our simulated shelf environments are viable for an ecophysiotype, or (equivalently) when $100 \%$ of metabolically viable habitat area is lost. Increasing the minimum number of inhabited ocean cells required to survive a warming event or decreasing the proportional habitat loss required for extinction moderately increases modeled global ecophysiotype extinction (Figs. S9-10). The differences in the shape of these responses highlight the importance of these parameters and the second- 
order effects of continental configuration. However, the overall trend of decreasing

233 extinction intensity with increased atmospheric $\mathrm{O}_{2}$, with an apparent inflection point

234 generally around $40 \%$ PAL, is consistent across all of these simulations. Similar trends

235 are observed when investigating the impacts of varying the distributions used to

236 parameterize physiological responses $\left(A_{\mathrm{o}}\right.$ - the inverse of the hypoxic threshold; and $E_{\mathrm{o}}-$

237 the temperature-dependency of the hypoxic threshold). While varying the distributions of

$238 A_{\mathrm{o}}$ and $E_{\mathrm{o}}$ does impact the magnitude of simulated global ecophysiotype extinction and

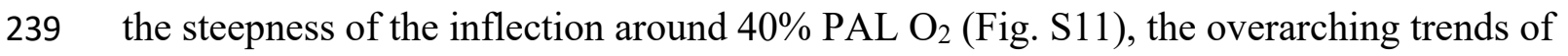

240 increasing predicted extinction with decreasing atmospheric oxygen (down to at least

$24120 \% \mathrm{PAL} \mathrm{O}_{2}$ ) are not impacted. The size of the synthetic population of ecophysiotypes

242 (Materials and Methods) has no noticeable effect on the analyses illustrated in Figure 5

243 (Fig. S12).

\section{Discussion}

246 Using a coupled Earth system and ecophysiological modeling approach, our analyses

247 suggest that oxygenation of the shallow ocean (as modulated by atmospheric oxygen

248 levels) is the dominant factor in governing the vulnerability of marine animals to

249 extinction during warming events. These results remain consistent when complex

250 oceanographic relationships between temperature and seawater oxygenation and

251 uncertainties in ocean circulation, biological pump efficiency, climate state, and habitat

252 area thresholds are taken into account. Critically, the relationship we observe between

253 simulated global ecophysiotype extinction and atmospheric oxygen is non-linear, with an

254 apparent inflection point around $40 \% \mathrm{PAL} \mathrm{O}_{2}$ (Fig. 5). This broadly coincides with the

255 upper limit of estimates for early Paleozoic atmospheric oxygen (Fig. 1; $(15,17)$ ),

256 supporting the hypothesis that relatively low atmospheric oxygen levels created boundary

257 conditions under which high-magnitude extinction events were more likely to occur as a

258 product of climate variability. 
While atmospheric oxygen is the primary control on extinction sensitivity in our simulations, our analyses show that other factors modulate the response to varying degrees. This is unsurprising considering how these factors affect the three-dimensional oxygen-temperature landscape of the ocean. In particular, our analyses indicate that continental configuration may play an important secondary role in governing ecophysiotype extinction vulnerability at the global scale (Fig. 5), although this is difficult to clearly separate from minor climatic differences between ensembles. Notably, we do not observe time-correlative trends between the three continental configurations compact continental configuration of the Ordovician is particularly different from that of

270 the more evenly latitudinally distributed and dispersed configuration of the Paleocene

271 when compared to the differences associated with changing atmospheric $\mathrm{O}_{2}$. The

272 efficiency of the biological pump (simulated here as a difference in prescribed

273 remineralization depths in the ocean) is also thought to have changed through the

274 Phanerozoic (17). However, we find that the differences in simulated extinction 275 sensitivity are minimal and (if anything) in opposition to the influence of changes in 276 atmospheric oxygenation (Fig. S7). This is likely because both continental configuration

277 and carbon remineralization depth mainly change oxygen distributions in the deeper 278 ocean, whereas most animals are living (and dying) in the surface ocean, which is closely 279 coupled to the $\mathrm{O}_{2}$ concentration of the atmosphere. Finally, while the habitat area 280 thresholds we used to define simulated extinction and specific parameterization of 281 physiological traits do impact the slope and magnitude of the observed relationship 282 between atmospheric oxygen and extinction (in expected directions; Figs. S9-11), the first 283 order trends remain consistent regardless of how we parametrize our ecophysiological 284 extinction model.

We additionally find that an important climate state-dependence exists for ecophysiological extinction sensitivity. Marine animals are expected to be living closer to

287 their thermal limits as defined by oxygen and capacity limited thermal tolerance in 288 warmer environments based on observations from modern tropical marine habitats (Fig. 
$2 ;(29,38))$, with implications for broader areas of the ocean in ancient greenhouse climates. We provide further model support for this hypothesis by demonstrating increased simulated extinction magnitudes in warmer climate states (Fig. S8). Very warm early Paleozoic sea surface temperatures have been reconstructed using geochemical paleothermometry (48-50), suggesting that this phenomenon could also provide some explanatory power for the frequency of high magnitude extinctions. However, while recent advances in paleothermometry have shown considerable potential in constraining Ordovician climate dynamics (50), there is still currently limited consensus on directional trends in early Phanerozoic sea surface temperatures, especially in the earliest Paleozoic (51-53). In contrast, reconstructions of atmospheric oxygen are increasingly consistent, even if the absolute concentrations are poorly constrained (Fig. 1). This discussion should not be framed as a question of oxygen versus temperature; inherent to the concept of temperature-dependent hypoxia is that both interact to determine aerobic safety margins (Fig. 2; $(29,34,38)$ ). We therefore suggest that atmospheric oxygen can confidently be established as a first-order control on early Phanerozoic extinction rates, and that potentially high sea surface temperatures through the same time interval may have amplified the effect of limited surface oxygenation. Changes in the latitudinal focus of fossil sampling through the Phanerozoic may also bias extinction rate estimates slightly towards environments with warmer baseline temperatures in the early Paleozoic when well-sampled paleocontinents were mainly situated at low latitudes (54). However, the lack of similarly high long-term extinction rates across other intervals with low-latitude sampling biases indicates that this cannot fully explain the trends we seek to explain here and that atmospheric oxygen (potentially compounded by warm global climate) is a more probable first-order driver.

The plausibility of temperature-dependent hypoxia as a driver of high early Paleozoic extinction rates is supported by links between sea-level changes, ocean deoxygenation and extinctions during this interval of geologic time. The CambrianOrdovician biomere-style extinction events are a major component of the characteristically high early Paleozoic extinction rates (9). These events, predominantly observed in the trilobite fossil record (e.g. (55)), have long been recognized as associated 
with sedimentological evidence of sea-level change, possible thermocline migration and ocean anoxia $(56,57)$. Stable isotope geochemistry provides further evidence that the biomere events were linked to major marine carbon and sulfur cycle perturbations, consistent with ocean warming and deoxygenation (58-61). Similar inferences of ocean anoxia and rapid sea-level change have been made for the early-middle Cambrian Botomian extinction event (62). In contrast, multiple Mesozoic and Cenozoic hyperthermals (including the Paleocene-Eocene Thermal Maximum and Cretaceous Oceanic Anoxic Event 2) that exhibit similar broad patterns of environmental change (43, 63-65) do not correlate with global marine extinctions of comparably high magnitude (Fig. 1; (9)). Our analyses demonstrate that the effects of changing atmospheric oxygenation on the thermal safety margins of marine ectotherms (Fig. 2) may account for this discrepancy in extinction magnitude between events with similar signatures of environmental change. Our simulations of metabolic habitat viability also offer a potential mechanism for the high origination rates observed through the early Paleozoic $(6,7)$ - particularly if simple logistic models of extinction and subsequent recovery (i.e. origination; e.g. $(4,66))$ can be broadly applied to the Phanerozoic fossil record. However, we focus upon warming driven extinction events because fewer assumptions regarding the evolutionary advantages of different ecophysiotypes (and how they relate to the rate at which physiological diversity increases in cool climate states) are required for these models, which simply simulate the instantaneous loss of viable ecophysiotypes.

Our combined Earth system and ecophysiological modeling approach provides a methodological advance in moving from simple correlation to more mechanistic understanding of how environmental change impacted marine ecosystems through Earth history. The use of physiology as a conceptual bridge between the fossil and geochemical records is of clear appeal for establishing a mechanistic understanding of animalenvironment interactions from the geologic record (67). However, the power of Earth system models in simulating the ecophysiological responses of marine animals to deeptime environmental change is only recently being explored (e.g. $(33,41))$. We apply simple assumptions about the diversification of ecophysiotypes (i.e. that ecophysiotypes proliferate in times of stability and become inviable during warming events) to test the 


\section{Conclusions}

376 Using a novel ensemble Earth system and ecophysiological modeling framework, we

hypothesis that limited atmospheric oxygenation would increase physiological vulnerability to ocean warming events on geologic timescales. For increasingly accurate models of animal-environment interactions through the Phanerozoic (for example, ones that directly simulate latitudinal gradients in taxonomic diversity), other key variables involved in these interactions will need to be incorporated. At the organism-scale, physiological traits including vulnerability to ocean acidification, food supply limitation and possible variations in physiological responses are all likely important variables. For example, the different buffering capacities of marine animal groups have previously been invoked to explain differential extinction selectivity $(68,69)$. Meta-analytical approaches also suggest that sensitivity to climate related stressors correlates with genus survival across ancient extinction events in higher-level taxonomic groups (34), further indicating that differences in physiological tolerances between marine clades may be an important consideration when applying ecophysiological principles to Earth history. While we demonstrate that our results are consistent across a range of deviations from mean modern physiological responses, it will hopefully become feasible to begin linking physiological traits more directly to extinct animal groups based on phylogenetic and adaptive principles as the availability of experimental respirometry data characterizing temperature-dependent hypoxic responses of marine ectotherms increases. At the macroecological scale, migration (as applied in Saupe et al. (41)), species interactions, and resource competition also play key roles in governing population and species responses to environmental change. As model frameworks for deep-time animalenvironment interactions develop in terms of both ecophysiological and biogeochemical realism, the temporal-spatial resolution of paleontological and geochemical databases will also be critical to the explanatory power of these methodologies, as will techniques addressing the limitations and biases inherent to the geologic record $(4-6,13,54,70-72)$.

\footnotetext{
demonstrate that atmospheric oxygen levels likely exerted a first-order control on
} 
extinction vulnerability through the Phanerozoic. Our model analyses illustrate that the theoretical predictions of oxygen and capacity limited thermal tolerance are expected to have globally significant implications, resulting in dramatically increased extinction of marine animals under varying climatic conditions during geological periods with limited surface oxygenation. While warmer initial climate states increase the magnitude of our simulated extinctions, and continental configuration and the strength of the biological pump also have minor impacts on our predictions, the trend of increased extinction vulnerability with early Paleozoic levels of atmospheric oxygen is dominant across all of our simulations. We therefore argue that the exceptional frequency of high-magnitude extinction events in the early Paleozoic was primarily a consequence of limited surface oxygenation and temperature-dependent hypoxia responses in marine animals.

\section{Materials and Methods}

\section{Quantitative paleobiology}

We performed a series of quantitative paleobiological analyses to evaluate evidence for declining extinction rates through the Phanerozoic. We adapted methods from Kocsis et al. (7) to generate new reconstructions and statistical analyses of extinction rates through the Phanerozoic specifically for marine ectotherms, using an updated download from the Paleobiology Database (PBDB) and omitting endothermic and/or exclusively airbreathing higher-level marine taxa (marine mammals, marine reptiles and turtles). We generated reconstructed extinction rates using three richness metrics (classical rarefaction, raw occurrences and shareholder quorum subsampling) and four extinction rate metrics (second-for-third substitution, corrected three-timer, gap-filler and percapita). We applied these metrics to generate reconstructions for all genera both at stage resolution and using the $\mathrm{PBDB} \sim 10$ million year bins. To investigate the potential impact of changing species to genus ratios through the Phanerozoic (e.g. (73)), we further conducted the same analyses on filtered datasets, generating reconstructions all genera, genera with a single accepted named species, genera with 2 species, genera with 3 
species, and genera with 4 or more accepted named species (Fig. S1). For shareholder quorum subsampling analyses we used a quorum level of 0.5 to accommodate lower sampling intensity in filtered datasets (as supported by Boag et al. (32)) and remain within the $>0.4$ quorum range suggested by previous authors (7). Figure 1 is a summary of the top-right panel of Figure S1, including all genera at stage resolution. We further fit cross-validated LOESS models to our reconstructed extinction rates to evaluate whether early Paleozoic decreases in extinction rates were a major feature of the full Phanerozoic record (Fig. S2). Finally, we generated Spearman's correlation coefficients to investigate the statistical significance of temporal declines in extinction rates across all of our treatments (Table S1). Adapted code from Kocsis et al. (7) and filtered Paleobiology Database download are available at https://github.com/richardstockey/cGENIE$\underline{\text { metabolic index.extinction and are assigned a DOI: } 10.5281 / \text { zenodo. } 5143583}$

\section{Earth system modeling}

cGENIE is an Earth system model of intermediate complexity, designed for the spatiallyexplicit biogeochemical characterization of global-scale paleoceanographic problems (39). We create a range of 3D realizations of ocean environmental conditions by running ensembles of model experiments. The experiments in each ensemble are run for 10,000 or 20,000 years (depending on the time required for dissolved marine $\mathrm{O}_{2}$ and seawater temperature to reach steady state), with a range of assumptions regarding atmospheric $p \mathrm{CO}_{2}$ specific to each continental configuration based upon changing solar luminosity and albedo (Fig. 3), and a range of atmospheric $p \mathrm{O}_{2}$ scenarios based upon the range of estimates from geochemical proxy data and biogeochemical box modeling (Fig. 1). We apply this ensemble treatment to three ancient continental configurations, chosen for their relatively even temporal spread across the Phanerozoic and history of use in previous paleoclimate studies - a Paleocene configuration ( $\sim 55 \mathrm{Ma})(43)$, a Permian configuration $(\sim 251 \mathrm{Ma})$ (74), and an Ordovician configuration ( 450 Ma) (based on the $8 \mathrm{PAL} \mathrm{CO}_{2}$ FOAM simulation of Pohl et al. (75) - see Supplementary Information). For each of these ancient configurations, we apply age-appropriate solar luminosity (76). Finally, in the 
435 Ordovician continental configuration, we explore the possible influence of postulated

436 Phanerozoic changes in organic matter remineralization depth in the ocean $(17,42)-$

437 testing $\sim 1 / 3$ modern, or specifically $200 \mathrm{~m}$, e-folding depth (42).

438 We extract the mean annual ocean temperature and ocean oxygen concentration

439 during the last simulated year for each ocean grid cell and generate corresponding

440 seawater oxygen partial pressure $\left(p \mathrm{O}_{2}\right)$ values (following the methods of Hofmann et al.

441 (77)). Changing solar luminosity and continental configuration results in different mean

442 sea surface temperatures for the same atmospheric $\mathrm{CO}_{2}$ assumption. We therefore

443 identify the $\mathrm{pCO}_{2}$ values for each continental configuration that create comparable

444 climate simulations (Table S2 and Supplementary Information). These climate scenarios

445 are compared using the mean equatorial $\left(3.2^{\circ} \mathrm{S}\right.$ to $3.2^{\circ} \mathrm{N}, 0-80 \mathrm{~m}$ depth) surface ocean

446 temperature for each model (Figs. 4, S4-6, Table S2).

447 The code for the version of the 'muffin' release of the cGENIE Earth system

448 model used in this paper is tagged as v0.9.19 and is assigned a DOI:

$44910.5281 /$ zenodo.4473048. Configuration files for the specific experiments presented in

450 the paper can be found in the directory: genie-userconfigs/MS/stockeyetal.PNAS.2021.

451 Details on the experiments, plus the command line needed to run each one, are given in

452 the readme.txt file in that directory. All other configuration files and boundary conditions

453 are provided as part of the code release.

454 A manual detailing code installation, basic model configuration, tutorials covering 455 various aspects of model configuration and experimental design, plus results output and 456 processing, is assigned a DOI: 10.5281/zenodo.4469678.

Ecophysiological simulations of animal-environment interactions

459 We combine our ensemble Earth system modeling experiments with a probabilistic 460 ecophysiological model of extinction vulnerability. The Metabolic Index, as defined by 461 Deutsch et al. (29) and Penn et al. (33), is configured here to calculate the proportion of 

responses) that can inhabit each ocean cell in the cGENIE Earth system model.

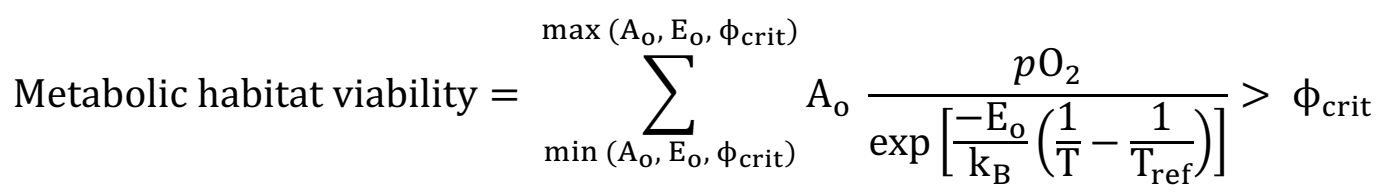

At the individual organism scale, $A_{\mathrm{o}}$ is the inverse of the hypoxic threshold of the organism (the minimum required seawater $p \mathrm{O}_{2}\left(P_{\text {crit }}\right)$ to sustain resting aerobic

468 metabolism), $E_{\mathrm{o}}$ is the temperature-dependency of the hypoxic threshold and $\phi_{\text {crit }}$ is the multiplicative increase in oxygen supply that is required to support ecologically

470 sustainable populations (calculated based on biogeographic distribution, relative to

471 experimental respirometry measurements of resting metabolism). In our modeling 472 approach, these variables are parametrized by probability density functions based on 473 observations from laboratory experiments and species distributions (Fig. 2; following 474 Penn et al. (33)). Seawater temperature and $\mathrm{pO}_{2}$ are environmental variables generated 475 through the Earth system modeling approach described above (Fig. 3). All other variables 476 are constant $\left(\mathrm{k}_{\mathrm{B}}\right.$ is the Boltzmann constant, $\mathrm{T}_{\text {ref }}$ is a reference temperature of $\left.15^{\circ} \mathrm{C}\right)$. In 477 our analyses, we simulate 1000 ecophysiotypes, sampled from probability distributions of $478 A_{\mathrm{o}}, E_{\mathrm{o}}$ and $\phi_{\text {crit }}$, and map which cells (if any) in each cGENIE ocean model are habitable 479 for each ecophysiotype (see Figure S12 for exploration of the number of ecotypes used).

Metabolic habitat viability, as defined here, quantifies the proportion of these 481 modeled ecophysiotypes that can live in the non-polar shelf environments (defined as any 482 cell adjacent to continental land mass in the top 3 layers of the cGENIE ocean, or $483<283.8 \mathrm{~m}$ water depth) of each global ocean model. See Figures S5-6 for sensitivity 484 analyses using the entire global ocean model, rather than shelf environments alone. Polar 485 environments $\left(>70^{\circ} \mathrm{N}\right.$ and $\left.<70^{\circ} \mathrm{S}\right)$ are excluded from all analyses due to the coarse model 486 resolution of polar ocean environments (e.g. in a Paleocene northern polar ocean) in 487 cGENIE and to reduce local non-linear responses to sea ice extent. 
We further simulate extinction as the global loss of ecophysiotypes between two

$489 \mathrm{CO}_{2}$ scenarios at the same $\mathrm{O}_{2}$ level. Specifically, we evaluate the proportional loss of

490 ecophysiotypes between baseline climate states (relatively cool, approximately pre-

491 industrial mean equatorial surface ocean temperatures - Supplementary Information) and

492 equivalent model scenarios that have undergone $\sim 5^{\circ} \mathrm{C}$ equatorial warming (Table S2;

493 modeling a nominal hyperthermal event of roughly the same magnitude as the Paleocene-

494 Eocene Thermal Maximum (43)) at each atmospheric $\mathrm{O}_{2}$ level. Global ecophysiotype

495 extinction is thus defined as the proportion of ecophysiotypes that were viable in shelf

496 environments of the colder baseline simulation but are no longer viable in any continental

497 shelf cell of the warmer ocean simulation. Sensitivity analyses exploring the number of

498 viable cells required to survive a simulated warming event, and the proportional habitat

499 loss required to drive extinction, are shown in Figures S9-10. Additional sensitivity

500 analyses exploring deviations from the distributions of $A_{\mathrm{o}}$ and $E_{\mathrm{o}}$ defined in Penn et al.

501 (33) are shown in Figure S11. In these analyses, "low" and "high" distributions have

502 mean values defined by the $25^{\text {th }}$ and $75^{\text {th }}$ percentiles of the distributions used in our

503 primary analyses, and half of the variance of those distributions. In plots of global

504 ecophysiotype extinction in shelf environments, we summarize the distribution of results

505 from 100 different simulated populations of 1000 randomly sampled ecophysiotypes to

506 illustrate the range of sampling-related uncertainty in our probabilistic approach to 507 ecophysiological modeling. All plots of single ecotype populations (i.e. those with points

508 rather than envelopes - Figs. 4, S4-6) use the same randomly sampled population.

509 R scripts to reproduce the ecophysiological analyses and associated figures are

510 available at https://github.com/richardstockey/cGENIE-metabolic index.extinction and

511 are assigned a DOI: $10.5281 /$ zenodo.5143583

\section{Acknowledgements}

513 We thank Thomas Boag and Justin Penn for helpful discussion. R.G.S. and E.A.S.

514 acknowledge support from National Science Foundation grant EAR1922966. R.G.S.

515 acknowledges support from the NASA Astrobiology Institute Early Career Collaboration

516 Award. A.R acknowledges support from the Heising-Simons Foundation. This project 
517 has received funding from the European Union's Horizon 2020 research and innovation 518 programme under the Marie Sklodowska-Curie grant agreement No. 838373. cGENIE 519 simulations were performed on Earth system modelling cluster facility at University of 520 California Riverside. Ecophysiological analyses of cGENIE models were performed on 521 the Sherlock cluster at Stanford University. We thank Stanford University and the 522 Stanford Research Computing Center for providing computational resources and support 523 that contributed to this research.

524

525

526

527 
1. D. M. Raup, J. J. Sepkoski, Mass extinctions in the marine fossil record. Science 215, 1501-1503 
in Oceanography and Marine Biology, An Annual Review, Volume 41, (CRC Press, 2003), pp. 145.

27. H. O. Pörtner, A. P. Farrell, Physiology and climate change. Science 322, 690-692 (2008).

28. H. O. Pörtner, Oxygen- And capacity-limitation of thermal tolerance: A matrix for integrating climate-related stressor effects in marine ecosystems. J. Exp. Biol. 213, 881-893 (2010).

29. C. Deutsch, A. Ferrel, B. Seibel, H. O. Pörtner, R. B. Huey, Climate change tightens a metabolic constraint on marine habitats. Science 348, 1132-1135 (2015).

30. E. M. Howard, et al., Climate-driven aerobic habitat loss in the California Current System. Sci. $A d v$. 6, eaay3188 (2020).

31. M. I. Duncan, N. C. James, W. M. Potts, A. E. Bates, Different drivers, common mechanism; the distribution of a reef fish is restricted by local-scale oxygen and temperature constraints on aerobic metabolism. Conserv. Physiol. 8 (2020).

32. T. H. Boag, W. Gearty, R. G. Stockey, Metabolic tradeoffs control biodiversity gradients through geological time. Curr. Biol. (2021) https:/doi.org/10.1016/j.cub.2021.04.021.

33. J. L. Penn, C. Deutsch, J. L. Payne, E. A. Sperling, Temperature-dependent hypoxia explains biogeography and severity of end-Permian marine mass extinction. Science 362, eaat1327 (2018).

34. C. J. Reddin, P. S. Nätscher, Á. T. Kocsis, H. O. Pörtner, W. Kiessling, Marine clade sensitivities to climate change conform across timescales. Nat. Clim. Chang. 10, 249-253 (2020).

35. T. H. Boag, R. G. Stockey, L. E. Elder, P. M. Hull, E. A. Sperling, Oxygen, temperature and the deep-marine stenothermal cradle of Ediacaran evolution. Proc. R. Soc. B Biol. Sci. 285, 20181724 (2018).

36. C. T. Reinhard, N. J. Planavsky, S. L. Olson, T. W. Lyons, D. H. Erwin, Earth's oxygen cycle and the evolution of animal life. Proc. Natl. Acad. Sci. 113, 8933-8938 (2016).

37. E. A. Sperling, A. H. Knoll, P. R. Girguis, The Ecological Physiology of Earth's Second Oxygen Revolution. Annu. Rev. Ecol. Evol. Syst. 46, 215-235 (2015).

38. C. Deutsch, J. L. Penn, B. Seibel, Metabolic trait diversity shapes marine biogeography. Nature 585, 557-562 (2020).

39. A. Ridgwell, et al., Marine geochemical data assimilation in an efficient Earth system model of global biogeochemical cycling. Biogeosciences 4, 87-104 (2007).

40. C. J. Reddin, Á. T. Kocsis, W. Kiessling, Climate change and the latitudinal selectivity of ancient marine extinctions. Paleobiology 45, 70-84 (2019).

41. E. E. Saupe, et al., Extinction intensity during Ordovician and Cenozoic glaciations explained by cooling and palaeogeography. Nat. Geosci. 13, 65-70 (2020).

42. K. M. Meyer, A. Ridgwell, J. L. Payne, The influence of the biological pump on ocean chemistry: implications for long-term trends in marine redox chemistry, the global carbon cycle, and marine animal ecosystems. Geobiology 14, 207-219 (2016).

43. M. Gutjahr, et al., Very large release of mostly volcanic carbon during the Palaeocene-Eocene Thermal Maximum. Nature 548, 573-577 (2017).

44. G. L. Foster, D. L. Royer, D. J. Lunt, Future climate forcing potentially without precedent in the last 420 million years. Nat. Commun. 8 (2017).

45. S. N. C. Woolley, et al., Deep-sea diversity patterns are shaped by energy availability. Nature 533, 393-396 (2016).

46. E. A. Sperling, R. G. Stockey, The temporal and environmental context of early animal evolution: Considering all the ingredients of an "explosion." Integr. Comp. Biol. 58, 605-622 (2018).

47. B. A. Seibel, C. Deutsch, Oxygen supply capacity in animals evolves to meet maximum demand at the current oxygen partial pressure regardless of size or temperature. J. Exp. Biol. 223, jeb.210492 (2020).

48. T. W. Hearing, et al., An early Cambrian greenhouse climate. Sci. Adv. 4, eaar5690 (2018).

49. J. A. Trotter, I. S. Williams, C. R. Barnes, C. Lecuyer, R. S. Nicoll, Did Cooling Oceans Trigger Ordovician Biodiversification? Evidence from Conodont Thermometry. Science 321, 550-554 (2008).

50. S. L. Goldberg, T. M. Present, S. Finnegan, K. D. Bergmann, A high-resolution record of early Paleozoic climate. Proc. Natl. Acad. Sci. 118, e2013083118 (2021).

51. C. Vérard, J. Veizer, On plate tectonics and ocean temperatures. Geology 47, 882-885 (2019). 
52. B. J. W. Mills, et al., Modelling the long-term carbon cycle, atmospheric CO2, and Earth surface temperature from late Neoproterozoic to present day. Gondwana Res. 67, 172-186 (2019).

53. K. D. Bergmann, et al., A paired apatite and calcite clumped isotope thermometry approach to estimating Cambro-Ordovician seawater temperatures and isotopic composition. Geochim. Cosmochim. Acta 224, 18-41 (2018).

54. R. A. Close, R. B. J. Benson, E. E. Saupe, M. E. Clapham, R. J. Butler, The spatial structure of Phanerozoic marine animal diversity. Science 368, 420-424 (2020).

55. A. R. Palmer, Trilobites of the Late Cambrian pterocephaliid biomere in the Great Basin, United States. U. S. Geol. Surv. Prof. Pap. 493, 1-104 (1965).

56. A. R. Palmer, The biomere problem: evolution of an idea. J. Paleontol. 58, 599-611 (1984).

57. R. A. Fortey, There are Extinctions and Extinctions: Examples from the Lower Palaeozoic. Philos. Trans. R. Soc. B Biol. Sci. 325, 327-355 (1989).

58. M. R. Saltzman, C. T. Edwards, J. M. Adrain, S. R. Westrop, Persistent oceanic anoxia and elevated extinction rates separate the Cambrian and Ordovician radiations. Geology 43, 807-810 (2015).

59. B. C. Gill, et al., Geochemical evidence for widespread euxinia in the Later Cambrian ocean. Nature 469, 80-83 (2011).

60. M. Elrick, S. Rieboldt, M. Saltzman, R. M. McKay, Oxygen-isotope trends and seawater temperature changes across the Late Cambrian Steptoean positive carbon-isotope excursion (SPICE event). Geology 39, 987-990 (2011).

61. M. A. LeRoy, B. C. Gill, E. A. Sperling, N. R. McKenzie, T.-Y. S. Park, Variable redox conditions as an evolutionary driver? A multi-basin comparison of redox in the middle and later Cambrian oceans (Drumian-Paibian). Palaeogeogr. Palaeoclimatol. Palaeoecol., 110209 (2021).

62. A. Y. Zhuravlev, R. A. Wood, Anoxia as the cause of the mid-Early Cambrian (Botomian) extinction event. Geology 24, 311-314 (1996).

63. W. Yao, A. Paytan, U. G. Wortmann, Large-scale ocean deoxygenation during the PaleoceneEocene thermal maximum. Science 361, 804-806 (2018).

64. H. C. Jenkyns, Geochemistry of oceanic anoxic events. Geochemistry, Geophys. Geosystems 11 (2010).

65. M. O. Clarkson, et al., Uranium isotope evidence for two episodes of deoxygenation during Oceanic Anoxic Event 2. Proc. Natl. Acad. Sci. 115, 201715278 (2018).

66. D. H. Erwin, Lessons from the past: Biotic recoveries from mass extinctions. Proc. Natl. Acad. Sci. U. S. A. 98, 5399-5403 (2001).

67. A. H. Knoll, Systems paleobiology. Bull. Geol. Soc. Am. 125, 3-13 (2013).

68. A. H. Knoll, R. K. Bambach, J. L. Payne, S. Pruss, W. W. Fischer, Paleophysiology and endPermian mass extinction. Earth Planet. Sci. Lett. 256, 295-313 (2007).

69. J. L. Payne, M. E. Clapham, End-permian mass extinction in the oceans: An ancient analog for the twenty-first century? Annu. Rev. Earth Planet. Sci. 40, 89-111 (2012).

70. J. X. Fan, et al., A high-resolution summary of Cambrian to early Triassic marine invertebrate biodiversity. Science 367, 272-277 (2020).

71. A. Mehra, et al., Curation and Analysis of Global Sedimentary Geochemical Data to Inform Earth History. GSA Today 31, 4-9 (2021).

72. Ú. C. Farrell, et al., The Sedimentary Geochemistry and Paleoenvironments Project. Geobiology, gbi.12462 (2021).

73. K. W. Flessa, D. Jablonski, Declining Phanerozoic background extinction rates: Effect of taxonomic structure? Nature 313, 216-218 (1985).

74. K. M. Meyer, L. R. Kump, A. Ridgwell, Biogeochemical controls on photic-zone euxinia during the end-Permian mass extinction. Geology 36, 747-750 (2008).

75. A. Pohl, et al., Glacial onset predated Late Ordovician climate cooling. Paleoceanography 31, 800821 (2016).

76. D. O. Gough, "Solar Interior Structure and Luminosity Variations" in Physics of Solar Variations, (Springer Netherlands, 1981), pp. 21-34.

77. A. F. Hofmann, E. T. Peltzer, P. M. Walz, P. G. Brewer, Hypoxia by degrees: Establishing definitions for a changing ocean. Deep. Res. Part I Oceanogr. Res. Pap. 58, 1212-1226 (2011). 
78. C. T. Edwards, M. R. Saltzman, D. L. Royer, D. A. Fike, Oxygenation as a driver of the Great

689 Ordovician Biodiversification Event. Nat. Geosci. 10, 925-929 (2017).

690

691 
692

Figures

693 

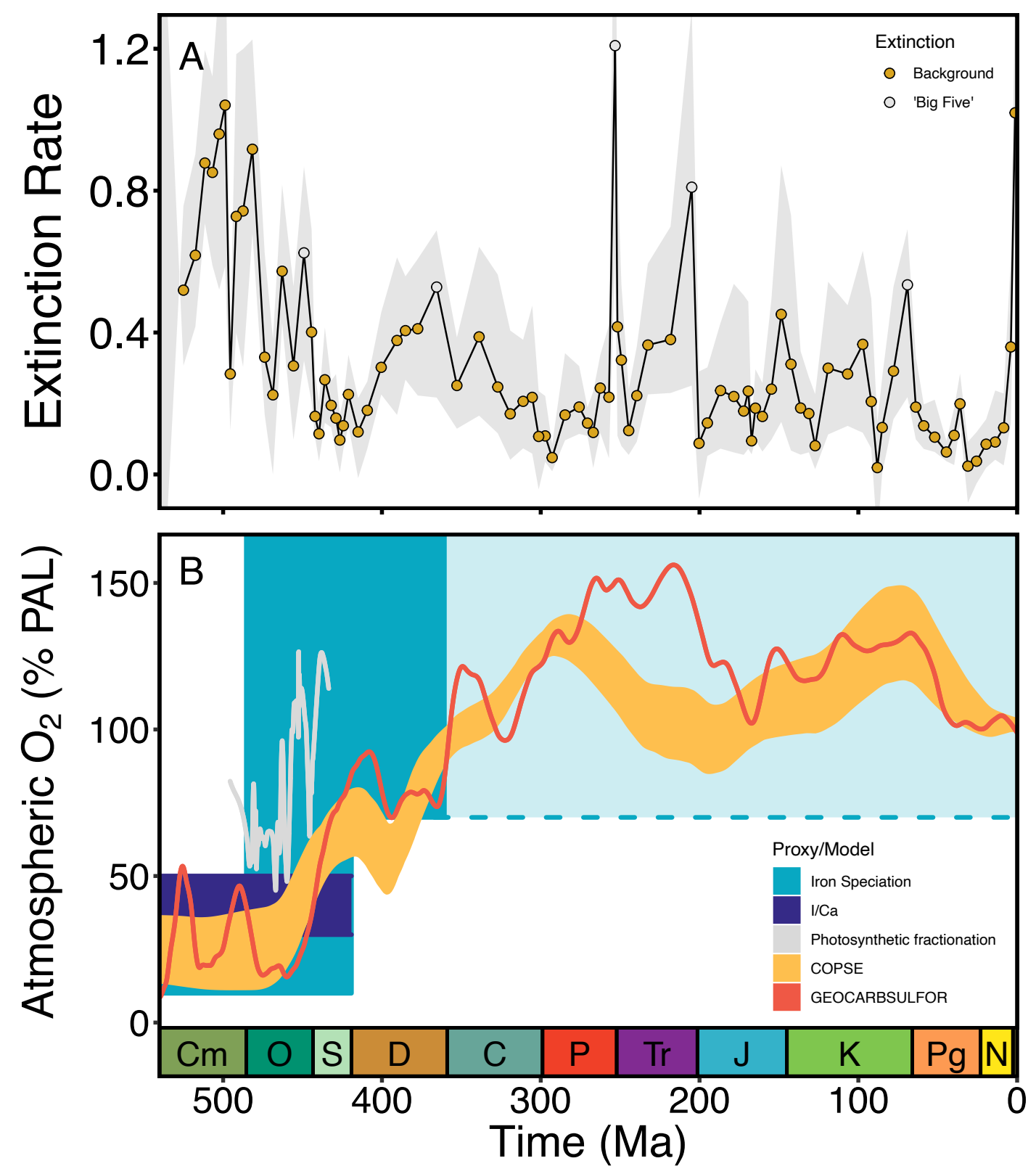

694

Figure 1: Trends in atmospheric oxygen and extinction rates through the Phanerozoic. A) Genus-level extinction rates for marine ectotherms (adapted from Kocsis et al. (7), Materials and Methods) - line and points represent mean values, envelope represents maximum and minimum estimates from all 12 methodological approaches (3 treatments: raw, classical rarefaction and Shareholder Quorum Subsampling-standardized, for each of 4 metrics: average per capita rates, corrected three-timer rates, gap-filler rates and second-for-third substitution rates). B) Reconstructions of atmospheric oxygen from biogeochemical models $(21,22,78)$ and geochemical proxy records $(15,17)$. 
Metabolic Index parameters:
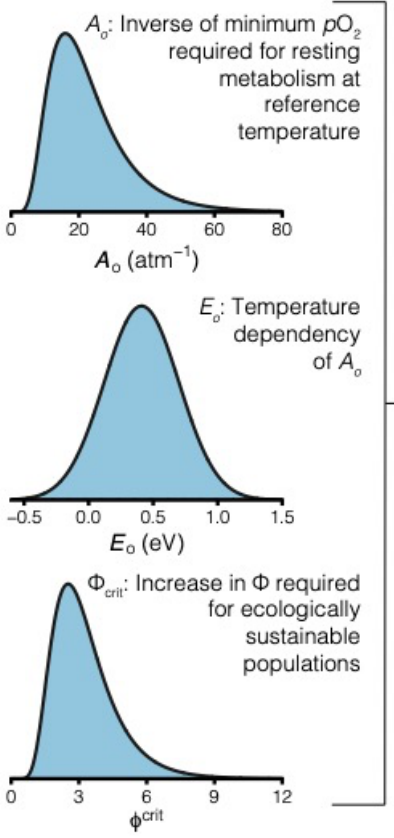

703

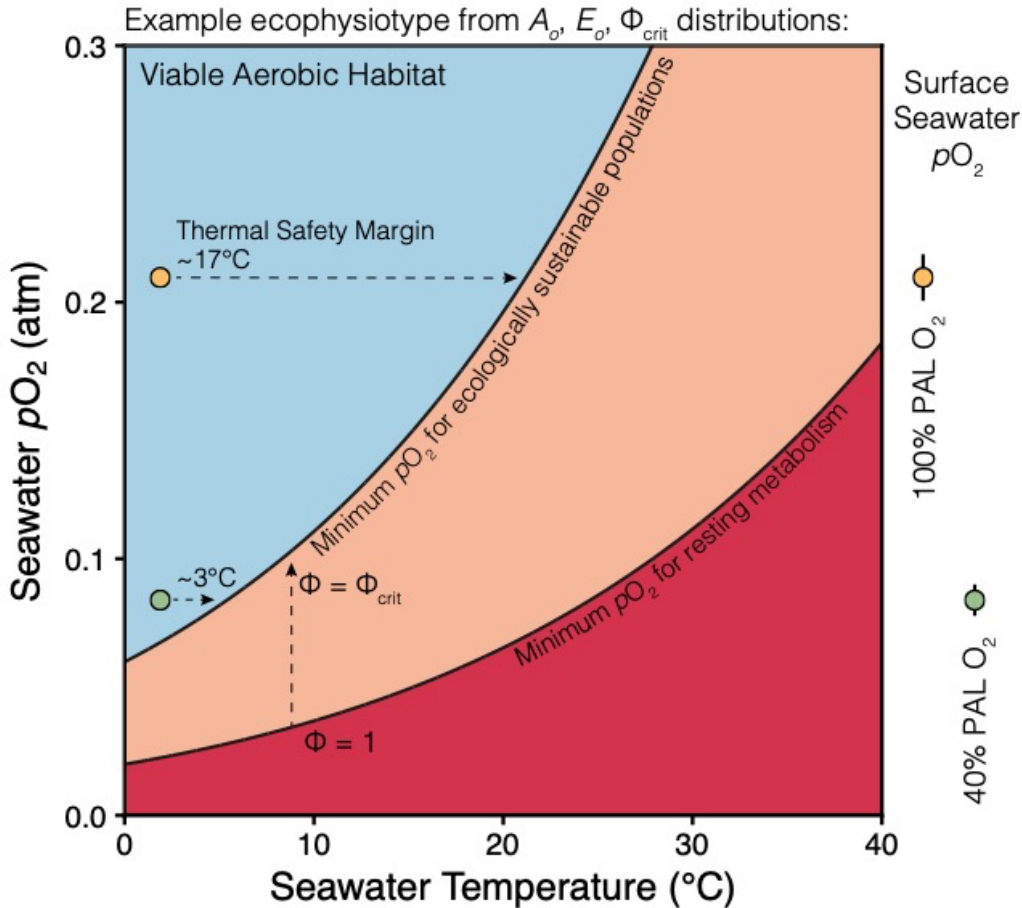

Figure 2: Schematic of ecophysiological model based on the Metabolic Index (Equation 1). The frequency distributions of three key ecophysiological parameters $-A_{0}, E_{\mathrm{o}}$ and $\phi_{\text {crit }}-$ are illustrated to the left. The implications of these parameters for the ecophysiological modeling framework employed in this study are illustrated for an example ecophysiotype on the right, based on the median values of these distributions. The minimum seawater $p \mathrm{O}_{2}$ required for resting metabolism increases at an exponential rate, primarily due to temperature effects on metabolic rate. The minimum seawater $p \mathrm{O}_{2}$ required for sustainable populations on ecological timescales also increases exponentially with temperature (faster than the minimum $p \mathrm{O}_{2}$ required for resting metabolism), as the product of an exponential function defined by $A_{\mathrm{o}}, E_{\mathrm{o}}$ and $\phi_{\text {crit }}$ (see Materials and Methods, Equation 1 for full details). At a given temperature, the thermal safety margin of a hypothetical ecophysiotype is predicted to decrease with decreasing seawater $p \mathrm{O}_{2}$ (either due to a change in equilibrium oxygen levels resulting from decreasing atmospheric oxygen, or a decrease in saturation resulting from biogeochemical processes such as organic carbon remineralization). Surface seawater $p \mathrm{O}_{2}$ distributions (far right) are taken from Earth system model analyses presented in this study (8 PAL $\mathrm{CO}_{2}$ Ordovician configuration; points represent mean values, and error bars $2 \mathrm{SD}$ ). 


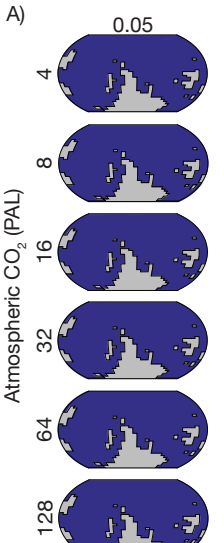

720

721

722

723

724

725

726

727

728

729

730

731
Atmospheric $\mathrm{O}_{2}$ (PAL)
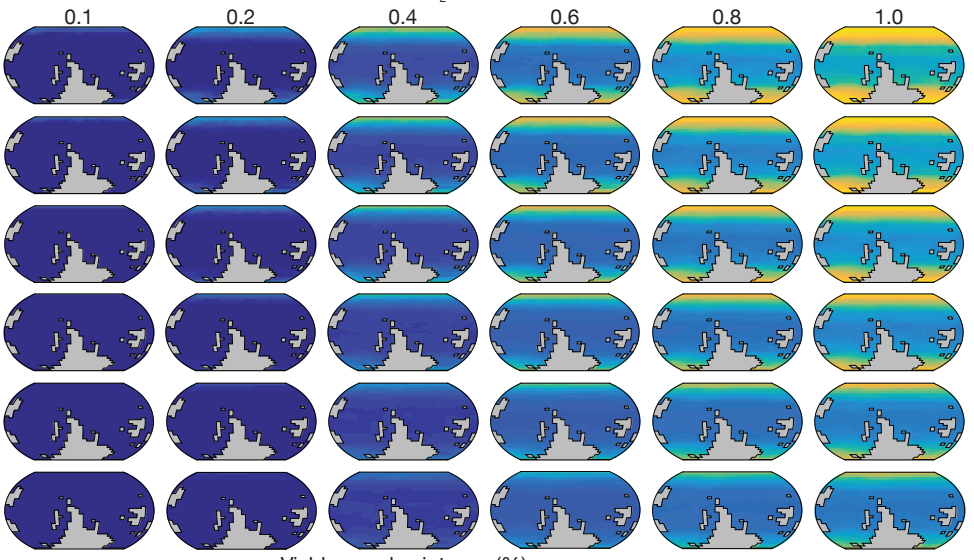

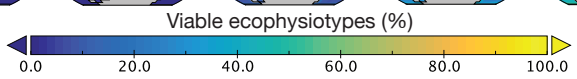

B) Ordovician
$\mathrm{CO}_{2}: 4,8,16,32,64,128$ PAL

$\mathrm{CO}_{2}: 4,8,16,32,64,128 \mathrm{PA}$
$(-3.79 \%$ solar luminosity $)$

Sensitivity analyses: remineralization depth

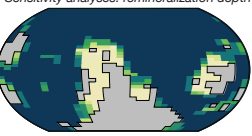

Permian
$\mathrm{CO}_{2}: 2,4,8,16,32,64$ PAL
$(2,114,5)$

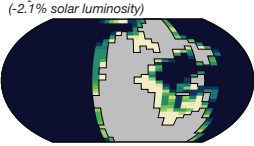

$\mathrm{CO}_{2}: 0.5,1,2,4,8,16 \mathrm{PAL}$

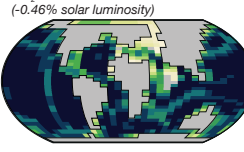

Ocean depth $(m)$

Figure 3: Ensemble Earth system modeling approach using cGENIE. A) Example of ensemble $\mathrm{O}_{2}-\mathrm{CO}_{2}$ Earth system modeling experiment using Ordovician continental configuration with modern remineralization depth. Color scale illustrates the proportion of the modeled ecophysiotypes that can live in each cell of the surface ocean (top 0-80m layer of the 16 layer cGENIE ocean is illustrated here). B) Sensitivity analyses performed in this study. Variations in continental configuration, remineralization depth and $\mathrm{CO}_{2}$ forcing are detailed here. The atmospheric $\mathrm{O}_{2}$ levels investigated in each ensemble experiment are the same. We present full surface ocean models here for ease of visual comparison, although main text analyses use only non-polar shelf environments (as illustrated in Fig. S3). 


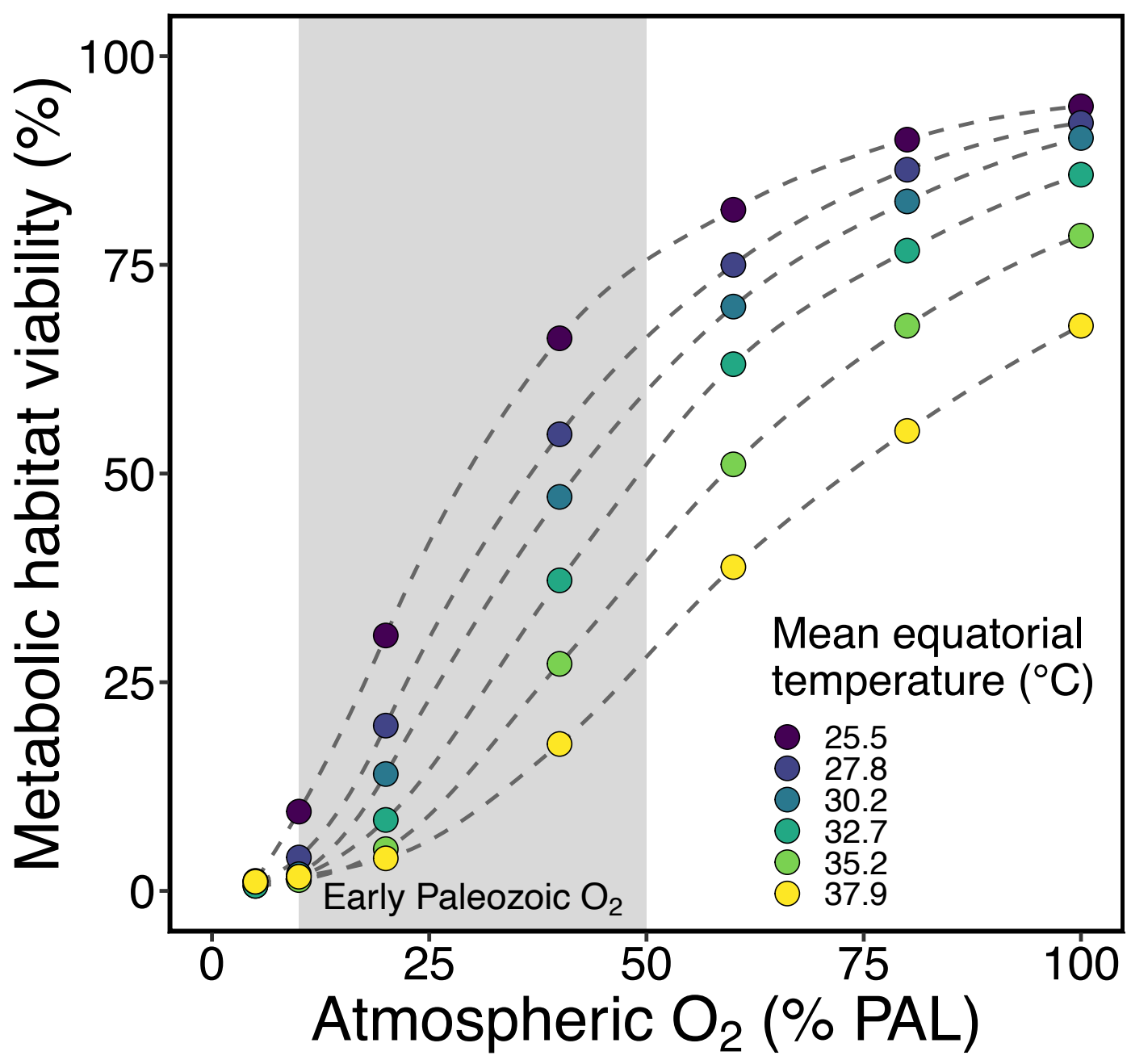

Figure 4: Metabolic habitat viability for the ensemble $\mathrm{O}_{2}-\mathrm{CO}_{2}$ Earth system modeling experiment with an Ordovician continental configuration and modern remineralization depth. Metabolic habitat viability is calculated as the proportion of the ecophysiotypes modeled that can live in shelf environments of each three dimensional ocean model produced using cGENIE (Materials and Methods). Circles represent individual experiments and are color coded by mean equatorial sea surface temperature $\left(-3.2^{\circ} \mathrm{S}\right.$ to $3.2^{\circ} \mathrm{N}, 0-80 \mathrm{~m}$ depth), as a product of atmospheric $\mathrm{CO}_{2}$ forcing. 


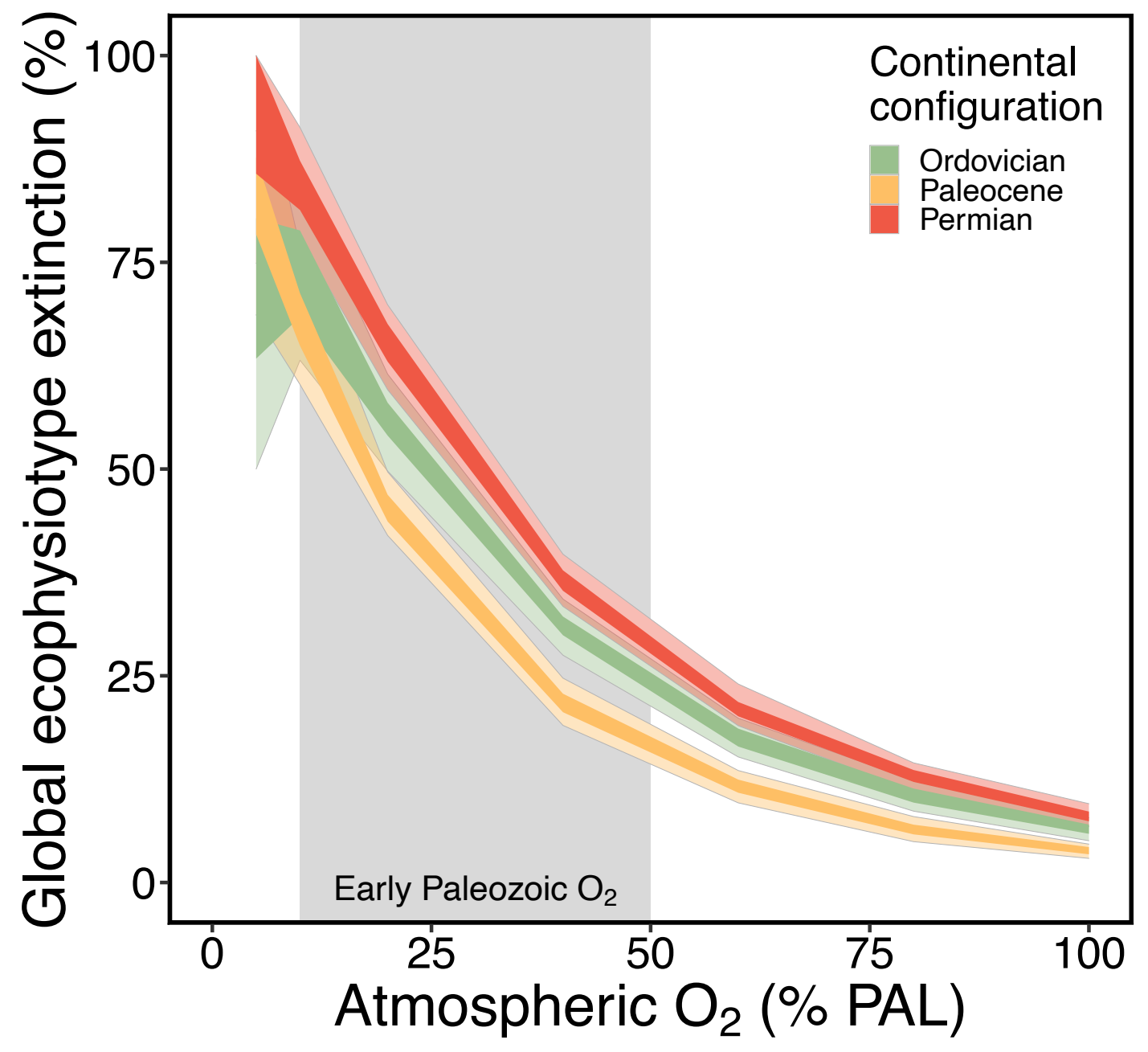

Figure 5: Global ecophysiotype extinction predicted to result from a $\sim 5^{\circ} \mathrm{C}$ warming event from an approximately pre-industrial baseline sea surface temperature at different atmospheric $\mathrm{O}_{2}$ levels, illustrating sensitivity analyses evaluating the influence of continental configuration on predicted extinction. Global ecophysiotype extinction is defined as the proportion of ecophysiotypes that were viable in the shelf environments of the colder baseline simulation but are no longer viable in any cell of the shelf environments of the warmer ocean simulation (Materials and Methods). Baseline $\mathrm{CO}_{2}$ levels used: 1 PAL - Paleocene; 8 PAL - Permian; 8 PAL - Ordovician. Darker envelopes represent $25^{\text {th }}-75^{\text {th }}$ percentiles of 100 simulation results, lighter envelopes represent $5^{\text {th }}-95^{\text {th }}$ percentiles. 